\title{
A Numerical Solution of Parabolic-Type Volterra Partial Integro-differential Equations by Laguerre Collocation Method
}

\author{
Burcu Gürbüz*, Mehmet Sezer \\ Department of Mathematics, Faculty of Art and Science, Celal Bayar University, 45140, Manisa, Turkey. \\ * Corresponding author. Tel.: +902362013202; email: burcugrbz@gmail.com \\ Manuscript submitted January 10, 2016; accepted March 8, 2016. \\ doi: 10.17706/ijapm.2017.7.1.49-58
}

\begin{abstract}
Partial integro-differential equations occur in many fields of science and engineering. Besides, the class of parabolic-type differential equations is modelled in compression of poro-viscoelastic media, reaction-diffusion problems and nuclear reactor dynamics. In recent years, most mathematical models used in many problems of physics, biology, chemistry and engineering are based on integral and integro-differential equations. In this work, we propose a new effective numerical scheme based on the Laguerre matrix-collocation method to obtain the approximate solution of one dimensional parabolic-type Volterra partial integro-differential equations with the initial and boundary conditions. The presented method reduces the solution of the mentioned partial integro-differential equation to the solution of a matrix equation corresponding to system of algebraic equations with unknown Laguerre coefficients. Also, some numerical examples together with error estimation are presented to illustrate the validity and applicability of the proposed scheme.
\end{abstract}

Key words: Laguerre series, laguerre matrix-collocation method, parabolic-type volterra partial integro-differential equations, error estimation.

\section{Introduction}

In this study, we consider parabolic-type Volterra partial integro-differential equations which combined the partial differentiations and the integral term. Partial integro-differential equation and its applications play an important role from biology to physics and engineering, and from economics to medicine. However, there are some different types of partial integro-differential equations and we focus on the parabolic-type of these equations [1]-[3].

This class of equations is applied in many different areas such as compression of convection-diffusion, reaction-diffusion problems and nuclear reactor dynamics. In particular, there are some numerical methods are useful to get approximate solutions; such as finite element methods, Rayleigh-Ritz, Galerkin, iterative methods, collocation methods and so on [4]. In this study, we develop an efficient Laguerre matrix-collocation method for solving the following parabolic-type Volterra partial integro-differential equation

$$
u_{t}(x, t)=g(x, t)+a(x, t) u_{x x}(x, t)+\int_{0}^{t} K(x, t, s) u(x, s) d s, 0 \leq x \leq l, 0 \leq t \leq T
$$


under the initial and boundary conditions

$$
\begin{gathered}
u(x, 0)=f(x), \quad x \in[0, l] \\
u_{t}(x, 0)=m(x), \quad x \in[0, l] \\
u(0, t)=h(t), \quad t \in[0, T]
\end{gathered}
$$

where $u_{t}=\partial u / \partial t, u_{x x}=\partial^{2} u / \partial x^{2}$. The functions $g, a$ are continuous functions and $K$ is the kernel function of the integral part of (1) which are define on $\mathbb{R}^{2}$ and $f, m, h$ are functions on $\mathbb{R}$ for our purpose, we assume the approximate solution of the problem (1) (2) in the truncated Laguerre series form

$$
u(x, t) \cong \sum_{n=0}^{N} \sum_{s=0}^{N} a_{n, s} L_{n, s}(x, t), \quad L_{n, s}(x, t)=L_{n}(x) L_{s}(t)
$$

where $L_{n}(x)$ denotes the Laguerre polynomials;

$$
L_{n}(x)=\sum_{k=0}^{n} \frac{(-1)^{k}}{k !}\left(\begin{array}{l}
n \\
k
\end{array}\right) x^{k}, n \in \mathrm{N}, 0 \leq x \leq \infty .
$$

and $a_{n, s},(n, s=0,1, \ldots, N)$ are unknown Laguerre polynomial coefficients, and $N$ is chosen as any positive integer such that $N \geq 2$.

The outline of this paper is as follows. In the next section we introduce some important properties of Laguerre polynomials. In Section 3, fundamental matrix relations and Laguerre matrix-collocation method is introduced. Section 4 discusses various techniques that can be used to perform error analysis. Section 5 is devoted to some illustrative examples of parabolic-type Volterra partial integro-differential equations on this topic. The paper concludes with a discussion of future developments [5], [6].

\section{Some Important Properties of Laguerre Polynomials}

Laguerre polynomials $L_{n}(x, \alpha)$ are orthogonal in the interval $(0,+\infty)$ with respect to the weight function $\omega(x, \alpha)=x^{\alpha} e^{-x}$. For $\alpha=0$, these polynomials become ordinary Laguerre polynomials $L_{n}(x)$; $L_{n}(x, 0)=L_{n}(x)$. Polynomials $L_{n}(x, \alpha)$ are defined by the generating function

$$
(1-t)^{-(\alpha+1)} \frac{e^{-x t}}{(1-t)}=\sum_{n=0}^{+\infty} L_{n}(x, \alpha) \frac{t^{n}}{n !}
$$

From the relation (5), the three term recurrence relation is obtained as

$$
(n+1) L_{n+1}(x, \alpha)=(2 n+\alpha+1-x) L_{n}(x, \alpha)-(n+\alpha) L_{n-1}(x, \alpha)
$$

with starting values $L_{0}(x, \alpha)=0, L_{1}(x, \alpha)=\alpha+1-x$. Expanding the left side of (5) in powers of $t$, and then comparing coefficients with $t^{n}$, the explicit representation of $L_{n}(x, \alpha)$ is obtained as

$$
\begin{gathered}
L_{n}(x, \alpha)=\sum_{k=0}^{n}(-1)^{n}\left(\begin{array}{l}
n \\
k
\end{array}\right) \frac{\Gamma(\alpha+n+1)}{\Gamma(\alpha+k+1)} \\
=\sum_{k=0}^{n}(-1)^{k}\left(\begin{array}{l}
n \\
k
\end{array}\right) \frac{(\alpha+n+1)_{n-k}}{\mathrm{k} !(n-k) !}
\end{gathered}
$$


where

$$
(s)_{n}=s(s+1) \ldots(s+n-1)=\frac{\Gamma(s+n)}{\Gamma(s)}
$$

and $\Gamma$ is the gamma function. Specifically, if $\alpha=0$, we can define (4) [7].

\section{Fundamental Matrix relations And Laguerre Matrix-Collocation Method}

Let us consider Eq. (1) and find the matrix forms of the equation. First we can write the solution function (3) in the matrix form

$$
[u(x, t)]=\mathbf{L}(x) \overline{\mathbf{L}}(t) \overline{\mathbf{A}}
$$

where

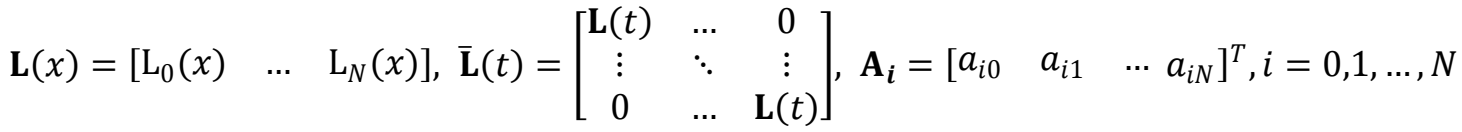

$$
\begin{aligned}
& \overline{\mathbf{A}}=\left[\begin{array}{lll}
\mathbf{A}_{0} & \ldots & \mathbf{A}_{N}
\end{array}\right]^{T}=\left[\begin{array}{llllllllllll}
a_{0,0} & a_{0,1} & \ldots & a_{0, N} & a_{1,0} & a_{1,1} & \ldots & a_{1, N} & \ldots & a_{N, 0} a_{N, 1} & \ldots & a_{N, N}
\end{array}\right]^{T}
\end{aligned}
$$

Then, we use the matrix relation

$$
\mathbf{L}(x)=\mathbf{X}(x) \mathbf{H}
$$

and

$$
\overline{\mathbf{L}}(t)=\overline{\mathbf{X}}(t) \overline{\mathbf{H}}
$$

where

$$
\begin{aligned}
& \mathbf{X}(x)=\left[\begin{array}{lll}
1 & x \ldots x^{N}
\end{array}\right], \quad \mathbf{X}(t)=\left[\begin{array}{lll}
1 & t \ldots t^{N}
\end{array}\right] \\
& \mathbf{H}=\left[\begin{array}{ccccc}
\frac{(-1)^{0}}{0 !}\left(\begin{array}{l}
0 \\
0
\end{array}\right) & \frac{(-1)^{0}}{0 !}\left(\begin{array}{l}
1 \\
0
\end{array}\right) & \frac{(-1)^{0}}{0 !}\left(\begin{array}{l}
2 \\
0
\end{array}\right) & \cdots & \frac{(-1)^{0}}{0 !}\left(\begin{array}{c}
N \\
0
\end{array}\right) \\
0 & \frac{(-1)^{1}}{1 !}\left(\begin{array}{l}
1 \\
1
\end{array}\right) & \frac{(-1)^{1}}{1 !}\left(\begin{array}{l}
2 \\
1
\end{array}\right) & \cdots & \frac{(-1)^{1}}{1 !}\left(\begin{array}{l}
N \\
1
\end{array}\right) \\
0 & 0 & \frac{(-1)^{2}}{2 !}\left(\begin{array}{l}
2 \\
2
\end{array}\right) & \cdots & \frac{(-1)^{2}}{2 !}\left(\begin{array}{c}
N \\
2
\end{array}\right) \\
\vdots & \vdots & \vdots & \ddots & \vdots \\
0 & 0 & 0 & \cdots & \frac{(-1)^{N}}{N !}\left(\begin{array}{l}
N \\
N
\end{array}\right)
\end{array}\right], \overline{\mathbf{H}}=\left[\begin{array}{cccc}
\mathbf{H} & 0 & \cdots & 0 \\
0 & \mathbf{H} & \cdots & 0 \\
\vdots & \vdots & \ddots & \vdots \\
0 & 0 & \cdots & \mathbf{H}
\end{array}\right], \overline{\mathbf{X}}(t)=\left[\begin{array}{cccc}
\mathbf{X}(t) & 0 & \cdots & 0 \\
0 & \mathbf{X}(t) & \cdots & 0 \\
\vdots & \vdots & \ddots & \vdots \\
0 & 0 & \cdots & \mathbf{X}(t)
\end{array}\right]
\end{aligned}
$$

Also, the relations between the matrices $\mathbf{X}(x), \overline{\mathbf{X}}(t)$ and their derivatives $\mathbf{X}^{\prime}(x), \mathbf{X}^{\prime \prime}(x)$ and $\overline{\mathbf{X}}^{\prime}(t)$, $\overline{\mathbf{X}}^{\prime \prime}(t)$ can be written as

$$
\mathbf{X}^{\prime}(x)=\mathbf{X}(x) \mathbf{B}, \quad \mathbf{X}^{\prime \prime}(x)=\mathbf{X}(x)(\mathbf{B})^{2}
$$




$$
\overline{\mathbf{X}}^{\prime}(t)=\overline{\mathbf{X}}(t) \overline{\mathbf{B}}, \quad \overline{\mathbf{X}}^{\prime \prime}(t)=\overline{\mathbf{X}}(t)(\overline{\mathbf{B}})^{2}
$$

where

$$
\mathbf{B}=\left[\begin{array}{ccccc}
0 & 1 & 0 & \ldots & 0 \\
0 & 0 & 2 & \ldots & 0 \\
\vdots & \vdots & \vdots & \ddots & \vdots \\
0 & 0 & 0 & N & 0 \\
0 & 0 & 0 & 0 & 0
\end{array}\right] \quad \overline{\mathbf{B}}=\left[\begin{array}{cccc}
\mathbf{B} & 0 & \cdots & 0 \\
0 & \mathbf{B} & \cdots & 0 \\
\vdots & \vdots & \ddots & \vdots \\
0 & 0 & \cdots & \mathbf{B}
\end{array}\right]
$$

Besides, we organize the derivatives of $u(x, t)$ with respect to (6), (7), (8), (9) and (10) in the matrix forms

$$
\begin{array}{r}
{\left[u_{x}(x, t)\right]=\mathbf{L}^{\prime}(x) \overline{\mathbf{L}}(t) \overline{\mathbf{A}}=\mathbf{X}(x) \mathbf{B} \mathbf{H} \overline{\mathbf{L}}(t) \overline{\mathbf{A}}} \\
{\left[u_{x x}(x, t)\right]=\mathbf{L}^{\prime \prime}(x) \overline{\mathbf{L}}(t) \overline{\mathbf{A}}=\mathbf{X}(x) \mathbf{B}^{2} \mathbf{H} \overline{\mathbf{L}}(t) \overline{\mathbf{A}}} \\
{\left[u_{t}(x, t)\right]=\mathbf{L}(x) \overline{\mathbf{L}}^{\prime}(t) \overline{\mathbf{A}}=\mathbf{X}(x) \mathbf{H} \overline{\mathbf{L}}^{\prime}(t) \overline{\mathbf{A}}}
\end{array}
$$

Now, we convert the kernel function $K(x, t, s)$ and the function $u(x, s)$ to the matrix forms in the integral part of Eq.(1), by means of the following procedure. Firstly, the function $K(x, t, s)$ can be expressed by the truncated Taylor series

$$
K(x, t, s)=\sum_{p=0}^{N} \sum_{q=0}^{N} \sum_{r=0}^{N} k_{p q r} x^{p} t^{q} s^{r}, \quad k_{p q r}=\frac{1}{p ! q ! r !} \frac{\partial^{p+q+r} K(0,0,0)}{\partial x^{p} \partial t^{q} \partial s^{r}}, K=\left[k_{p q r}\right], p, q, r=0,1, \ldots, N
$$

and then, it can be written in the matrix form

$$
[K(x, t, s)]=\mathbf{X}(x) \overline{\mathbf{X}}(t) \mathbf{K} \mathbf{X}^{T}(s) .
$$

In addition, matrix form of the function $u(x, s)$ becomes from (6) and (7)

$$
[u(x, s)]=\mathbf{X}(x) \overline{\mathbf{X}}(s) \mathbf{A}=\mathbf{X}(s) \overline{\mathbf{X}}(x) \overline{\mathbf{A}} .
$$

By using (12) and (13), the matrix form of integral part are obtained as follows:

$$
\begin{aligned}
\int_{0}^{t} K(x, t, s) u(x, s) d s & =\int_{0}^{t} \mathbf{X}(x) \overline{\mathbf{X}}(t) \mathbf{K} \mathbf{X}^{T}(s) \mathbf{X}(s) \overline{\mathbf{X}}(x) \overline{\mathbf{A}}^{*} d s \\
& =\mathbf{X}(x) \overline{\mathbf{X}}(t) \mathbf{K}\left(\int_{0}^{t} \mathbf{X}^{T}(s) \mathbf{X}(s) d s\right) \overline{\mathbf{X}}(x) \overline{\mathbf{A}}^{*} \\
& =\mathbf{X}(x) \overline{\mathbf{X}}(t) \mathbf{K} \mathbf{Q}(t) \overline{\mathbf{X}}(x) \overline{\mathbf{A}}^{*}
\end{aligned}
$$

where

$$
\mathbf{Q}(t)=\left[q_{m n}(t)\right] \quad m, n=0,1,2 . \quad q_{m n}(t)=\frac{t^{m+n+1}}{m+n+1}
$$


By substituting the relations (11) and (14) into Eq.(1), we have the matrix form of (1)

$$
\left\{\mathbf{X}(x) \mathbf{H} \overline{\mathbf{L}}^{\prime}(t)-[a(x, t)] \mathbf{X}(x) \mathbf{B}^{2} \mathbf{H} \overline{\mathbf{L}}(t)\right\} \overline{\mathbf{A}}-\{\mathbf{X}(x) \overline{\mathbf{X}}(t) \mathbf{K} \mathbf{Q}(t) \overline{\mathbf{X}}(x)\} \overline{\mathbf{A}}^{*}=[g(x, t)]
$$

where

$$
\overline{\mathbf{A}}^{*}=\left[\begin{array}{lllll}
\overline{\mathbf{A}}_{0} & \overline{\mathbf{A}}_{1} & \ldots & \overline{\mathbf{A}}_{N}
\end{array}\right]^{T} \quad \mathbf{A}_{\boldsymbol{i}}^{*}=\left[\begin{array}{llll}
a_{0 i} & a_{1 i} & \ldots & a_{N i}
\end{array}\right]^{T}, i=0,1, \ldots, N
$$

Similarly, we organize the matrix equations for the initial and boundary conditions (2) by using (11):

$$
\begin{gathered}
{[u(x, 0)]=\mathbf{L}(x) \overline{\mathbf{L}}(0) \overline{\mathbf{A}}=\mathbf{X}(x) \mathbf{H} \overline{\mathbf{L}}(0) \overline{\mathbf{A}}=[f(x)]=\lambda} \\
{\left[u_{t}(x, 0)\right]=\mathbf{L}(x) \overline{\mathbf{L}}^{\prime}(0) \overline{\mathbf{A}}=\mathbf{X}(x) \mathbf{H}^{\prime}(0) \overline{\mathbf{A}}=[m(x)]=\mu} \\
{[u(0, t)]=\mathbf{L}(0) \overline{\mathbf{L}}(t) \overline{\mathbf{A}}=[h(t)]=\gamma}
\end{gathered}
$$

Then, we have the modified matrix system by simplifying (16)

$$
\mathbf{H} \overline{\mathbf{L}}(0) \overline{\mathbf{A}}=\lambda, \quad \mathbf{H} \overline{\mathbf{L}}^{\prime}(0) \overline{\mathbf{A}}=\mu, \quad \mathbf{L}(0) \overline{\mathbf{L}}(t) \overline{\mathbf{A}}=\gamma
$$

By putting the collocation points

$$
x_{i}=\frac{l}{N} i, i=0,1, \ldots, N, t_{j}=\frac{T}{N} j, j=0,1, \ldots, N \quad \text { and } h=\frac{l}{N}
$$

into Eq.(15), we have the fundamental matrix equation as

$$
\left\{\mathbf{X}(x) \mathbf{H} \overline{\mathbf{L}}^{\prime}\left(t_{j}\right)-\left[a\left(x_{i}, t_{j}\right)\right] \mathbf{X}\left(x_{i}\right) \mathbf{B}^{2} \mathbf{H} \overline{\mathbf{L}}\left(t_{j}\right)\right\} \overline{\mathbf{A}}-\left\{\mathbf{X}\left(x_{i}\right) \overline{\mathbf{X}}\left(t_{j}\right) \mathbf{K} \mathbf{Q}\left(t_{i}\right) \overline{\mathbf{X}}\left(x_{i}\right)\right\} \overline{\mathbf{A}}^{*}=\left[g\left(x_{i}, t_{j}\right)\right]=\mathbf{G}
$$

where

$$
\mathbf{G}=\left[\begin{array}{llllllllllll}
g_{0,0} & g_{0,1} & \ldots & g_{0, N} & g_{1,0} & g_{1,1} & \ldots & g_{1, N} & \ldots & g_{N, 0} g_{N, 1} & \ldots & g_{N, N}
\end{array}\right]^{T}
$$

Briefly,

$$
\mathbf{W}\left(x_{i}, t_{j}\right) \overline{\mathbf{A}}-\mathbf{W}^{*}\left(x_{i}, t_{j}\right) \overline{\mathbf{A}}^{*}=\mathbf{G}
$$

If we follow the same procedure for the initial and boundary conditions (17), we have,

$$
\mathbf{U} \overline{\mathbf{A}}=\lambda_{i}, \quad \mathbf{V} \overline{\mathbf{A}}=\mu_{i}, \quad \mathbf{Z} \overline{\mathbf{A}}=\gamma_{j}
$$

Consequently, to obtain the solution of Eq. (1) under the conditions (2), by replacing the row matrices (20) by the last rows of the augmented matrix (19), we have the required augmented matrix. Then, (19) becomes 


$$
\left[\tilde{\mathbf{W}} ; \tilde{\mathbf{W}}^{*} ; \mathbf{G}\right]
$$

By solving the augmented matrix form system the unknown Laguerre coefficients are computed. Thus, the approximate solution $u(x, t), 0 \leq x \leq l, 0 \leq t \leq T$ is found in the truncated Laguerre series (3), [8], [9].

\section{Error Analysis}

In this section, we give brief error estimation for the Laguerre polynomial solution (3) and it supports the accuracy of Laguerre polynomial solution. We define error function $x=x_{\alpha}, t=t_{\beta} \in[l, 0] \times[0, T], \alpha, \beta=0,1, \ldots$

$$
E_{N}\left(x_{\alpha}, t_{\beta}\right)=\left|u_{t}\left(x_{\alpha}, t_{\beta}\right)-a\left(x_{\alpha}, t_{\beta}\right) u_{x x}\left(x_{\alpha}, t_{\beta}\right)-\int_{0}^{t} K\left(x_{\alpha}, t_{\beta}, s\right) u\left(x_{\alpha}, s\right) d s-g\left(x_{\alpha}, t\right)\right| \cong 0,
$$

where $E_{N}\left(x_{\alpha}, t_{\beta}\right) \leq 10^{-k_{\alpha \beta}}=10^{-k}$, ( $k$ is positive integer) is prescribed, then the truncation limit $N$ increased until difference $E_{N}\left(x_{\alpha}, t_{\beta}\right)$ at each of the points becomes smaller than the prescribed $10^{-k}$. On the other hand we use different error norms for measuring errors. These error norms are defined as follows:

1) $L_{2}-E_{N}\left(x_{\alpha}, t_{\beta}\right)=\left(\sum_{i=1}^{n}\left(e_{i}\right)^{2}\right)^{1 / 2}$

2) $L_{\infty}-E_{N}\left(x_{\alpha}, t_{\beta}\right)=\operatorname{Max}\left(e_{i}\right), 0 \leq i \leq n$

3) $R M S-E_{N}\left(x_{\alpha}, t_{\beta}\right)=\sqrt{\frac{\sum_{i=1}^{n}\left(e_{i}\right)^{2}}{n+1}}$

where $e_{i}=u\left(x_{i}, \tau\right)-\hat{u}\left(x_{i}, \tau\right)$ also $u$ and $\hat{u}$ are the exact and approximate solutions of the problem respectively and $\tau$ is an arbitrary time $t$ in $[0, T][10]$.

\section{Illustrative Examples}

In this section, several numerical examples are given to illustrate the accuracy and effectiveness properties of the method and all of them were performed on the computer using a program written in Maple 18.

\subsection{Example 5.1}

We consider the parabolic-type Volterra partial integro-differential equation

$$
u_{t}(x, t)=g(x, t)+(x-5 t) u_{x x}(x, t)+\int_{0}^{t} 2 x s(t+1) u(x, s) d s, 0 \leq x \leq 1,0 \leq t \leq 1
$$

with following initial and boundary conditions

$$
\begin{aligned}
& u(x, 0)=u_{t}(x, 0)=0, \quad x \in[0,1] \\
& u(0, t)=-4 t^{2}, \quad t \in[0,1]
\end{aligned}
$$

where $g(x, t)=t\left(-3 t^{4} x^{4}+2 t^{4} x-3 t^{3} x^{4}+2 t^{3} x+9 t^{2} x-18 t x^{2}+6 x^{3}-8\right)$. We follow the same procedure in Section 
3 and by substituting (23) initial and boundary conditions the exact solution of (22) is obtained as $u(x, t)=\left(3 x^{3}-4\right) t^{2}$.

\subsection{Example 5.2}

We consider the following problem

$$
u_{t}(x, t)=u_{x x}(x, t)+\int_{0}^{t} u(x, s) d s+g(x, t) 0 \leq x \leq 1,0 \leq t \leq 1
$$

with initial and boundary conditions

$$
\begin{aligned}
& u(x, 0)=\left[\frac{r+1}{2}\right]\left(1-x^{6}\right), \quad x \in[0,1], r \leq 1, \\
& u(0, t)=u(1, t)=0, \quad t \in[0,1]
\end{aligned}
$$

where $g(x, t)$ is chosen with respect to $r$ value of exact solution $u(x, t)=\left[\frac{r+1}{2}\right]\left[\left(1-x^{6}\right) \sin (x+t)\right]$. Similarly, we have the approximate solution of (24) under the conditions (25). In Figure 1 and Figure 2, we can see the relation of the solutions for different $r$ values.

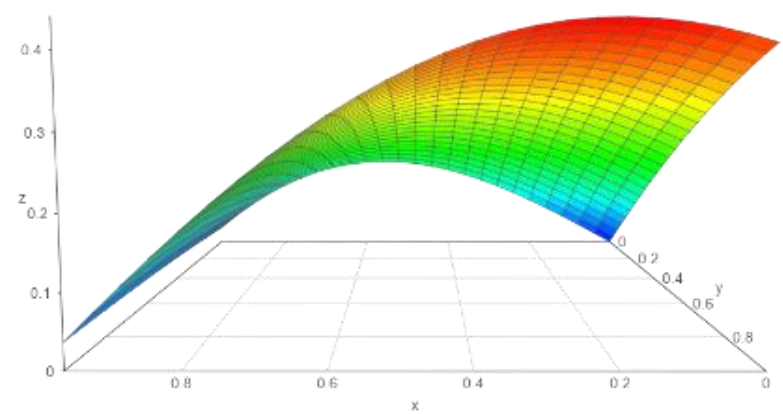

Fig. 1. Approximate solution for $\mathrm{r}=0$ value Example 5.2.

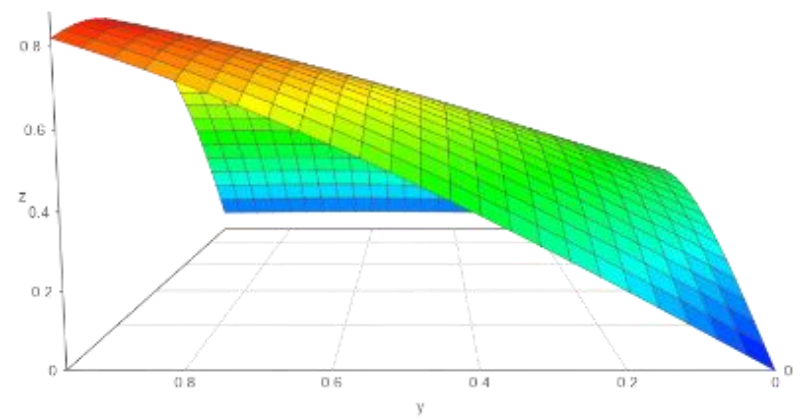

Fig. 2. Approximate solution for $r=1$ value Example 5.2.

\subsection{Example 5.3}

We consider the following problem

$$
u_{t}(x, t)=4 u_{x x}(x, t)+\int_{0}^{t} 3 t^{3} x^{2} u(x, s) d s+g(x, t) 0 \leq x \leq 1,0 \leq t \leq 1
$$


with initial and boundary conditions

$$
\begin{aligned}
& u(x, 0)=\cos \left(\pi x-\frac{\pi}{2}\right), \quad x \in[0,1] \\
& u(0, t)=u(1, t)=0, \quad t>0
\end{aligned}
$$

where $g(x, t)=5 x+\frac{1}{12} x^{3} t^{2}$. Similarly, we have the approximate solution of (26) under the conditions (27). In Table 2, we see that absolute, $L_{2}, L_{\infty}$ and RMS errors are examined.

Table 2. Absolute, $L_{2}, L_{\infty}$, RMS Errors of Example 5.3. for $\mathrm{N}=4$ and $\mathrm{h}=0.1$.

\begin{tabular}{lcccc}
\hline \hline t) & Absolute error & $L_{2}$-error & $L_{\infty}$-error & RMS-error \\
\hline 0.0 & $0.21205 \mathrm{E}-5$ & $0.25986 \mathrm{E}-7$ & $0.41187 \mathrm{E}-6$ & $0.23614 \mathrm{E}-5$ \\
\hline 0.1 & $0.35498 \mathrm{E}-4$ & $0.20562 \mathrm{E}-5$ & $0.77451 \mathrm{E}-5$ & $0.11033 \mathrm{E}-4$ \\
\hline 0.2 & $0.84625 \mathrm{E}-4$ & $0.35622 \mathrm{E}-5$ & $0.41422 \mathrm{E}-5$ & $0.22603 \mathrm{E}-4$ \\
\hline 0.3 & $0.75452 \mathrm{E}-4$ & $0.95136 \mathrm{E}-5$ & $0.45512 \mathrm{E}-4$ & $0.22603 \mathrm{E}-4$ \\
\hline 0.4 & $0.62451 \mathrm{E}-4$ & $0.75451 \mathrm{E}-4$ & $0.32134 \mathrm{E}-4$ & $0.32400 \mathrm{E}-4$ \\
\hline 0.5 & $0.95175 \mathrm{E}-4$ & $0.54247 \mathrm{E}-4$ & $0.65196 \mathrm{E}-4$ & $0.63541 \mathrm{E}-4$ \\
\hline 0.6 & $0.52533 \mathrm{E}-4$ & $0.55405 \mathrm{E}-4$ & $0.74157 \mathrm{E}-4$ & $0.78642 \mathrm{E}-4$ \\
\hline 0.7 & $0.65161 \mathrm{E}-4$ & $0.62142 \mathrm{E}-4$ & $0.54335 \mathrm{E}-4$ & $0.29413 \mathrm{E}-4$ \\
\hline 0.8 & $0.45261 \mathrm{E}-3$ & $0.95151 \mathrm{E}-4$ & $0.41142 \mathrm{E}-3$ & $0.06532 \mathrm{E}-3$ \\
\hline 0.9 & $0.85246 \mathrm{E}-3$ & $0.75481 \mathrm{E}-4$ & $0.21332 \mathrm{E}-3$ & $0.16273 \mathrm{E}-3$ \\
\hline 1.0 & $0.75395 \mathrm{E}-3$ & $0.74114 \mathrm{E}-4$ & $0.73194 \mathrm{E}-3$ & $0.35022 \mathrm{E}-3$ \\
\hline \hline
\end{tabular}

\section{Conclusion}

Parabolic-type Volterra partial integro-differential equations play an important role in the fields of science and engineering and numerical methods may be required to obtain their approximate solutions. Thus, the Laguerre collocation method has been presented. A considerable advantage of the method is shorter computation time and lower operation count results in reduction of cumulative truncation errors and improvement of overall accuracy. Illustrative examples support the efficiency of the method, and performed on the computer using a program written in Maple18 to obtain the results fast and reliable. As a result, the power of the employed method is confirmed. The method can also be extended to another applications but some modifications are required [11], [12].

\section{Acknowledgment}

This work is funded by Manisa Celal Bayar University Department of Scientific Research Projects, with grant ref. 2014-151 and performed within the "Numerical Solutions of Partial Functional Integro Differential Equations based on Laguerre Polynomials and Its Applications" project, Copyright 2016. All rights reserved.

\section{References}

[1] Agarwal, R. P. (1993). Contributions in Numerical Mathematics. Singapore: World Scientific Publishing.

[2] Dieudonné, J. (1985). Orthogonal Polynomials and Applications. Berlin-New York: Springer.

[3] Gürbüz, B., \& Sezer, M. (2014). Laguerre polynomial approach for solving Lane-Emden type functional differential equations. Journal of Applied Mathematics and Computation, 242, 255-264.

[4] Avazzadeh, Z., Beygi, R. Z., Maalek, G. F. M., \& Loghmani, G. B. (2012). A numerical solution of nonlinear parabolic-type Volterra partial integro-differential equations using radial basis functions. Journal of Engineering Analysis with Boundary Elements, 36, 881-893. 
[5] Kyrychko, Y. N., \& Hogan, S. J., (2010). On the use of delay equations in engineering applications. Journal of Vibration and Control, 16(7-8), 943-960.

[6] Jalilian, R., \& Hatami, F., (2012). A collocation method for the solution of convection-diffusion parabolic problems. Journal of Information and Computing Science, 7(3), 172-181.

[7] Aizenshtadt, V. S., \& Krylov, V. I. (1966). Tables of Laguerre Polynomials and Functions. Poland: Pergamon Press.

[8] Bülbül, B., \& Sezer, M. (2011). Taylor polynomial solution of hyperbolic type partial differential equations with constant coefficients. International Journal of Computer Mathematics, 88(3), 533-544.

[9] Kurt, N., \& Sezer, M. (2006). Solution of Dirichlet problem for a triangle region in terms of elliptic functions. Applied Mathematics and Computation, 182(1), 73-81.

[10] Bülbül, B., \& Sezer, M. (2013). A new approach to numerical solution of nonlinear Klein-Gordon equation. Mathematical Problems in Engineering, 7, 869749.

[11] Salahshour, S., Khajehnasiri, A. A., Safavi M., Ahmadian, A., \& Senu N. Approximate solutions of parabolic-type Volterra partial integro-differential equations with uncertainty.

[12] Gürbüz, B., \& Sezer, M. (2016). Numerical solutions of one-dimensional parabolic convection-diffusion problems arising in biology by the Laguerre collocation method. In Biomath Communications, 3(1).

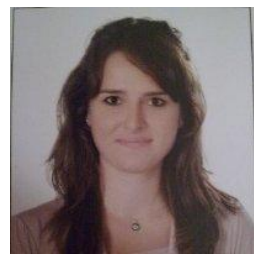

Burcu Gürbüz was born on August 13 1986 in Izmir, Turkey. She received her B.Sc. from the Department of Mathematics of Celal Bayar University in 2008. Her M.Sc. was in applied mathematics from Muğla Sitkı Koçman University in 2012. She has continued as PhD. student degree under the supervision of Prof. Dr. Mehmet Sezer in applied mathematics at the Celal Bayar University since 2013.

She was investigator funded by Fundação para a Ciência e a Tecnologia "Numerical simulation of three dimensional polymer flow and application to plastic forming" with reference PTDC/MAT/121185/2010 in University of Minho, Guimarães, Portugal, in 2012-2013. "Polynomial solutions of a class of nonlinear initial and boundary value problems arising in science and engineering fields" with reference 2014-148 project was funded by Department of Scientific Research Projects of Celal Bayar University and she was responsible researcher of the project. She has been part-time lecturer in Department of Engineering of Celal Bayar University and has been a researcher in Department of Scientific Research Projects of Celal Bayar University in "Numerical Solutions of Partial Functional Integro Differantial Equations with respect to Laguerre Polynomials and Its Applications” with reference 2014-151 project since 2014.

Her main research interests ordinary and partial differential equations, integral and integro differential-difference equations and scientific computation. Ms. Gürbüz is a member of Turkish Math Society (TMD) and European Society for Mathematical and Theoretical Biology (ESMTB).

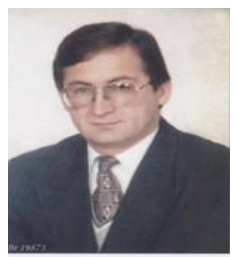

Mehmet Sezer was born on March 20, 1954 in Antalya, Turkey. He received his B.Sc. from the Department of Mathematics of Ege University in 1976 and was research assistant at Balıkesir Engineering Academy. His M.Sc. was from Ege University in 1977. He received his Ph.D. under the supervision of Prof. Dr. Süeda MORALI in the Department of Applied Mathematics at Ege University in 1982 and studied in the field of "Parabolic partial differential equations".

In 1982, he was Assistant Professor at Faculty of Education (Ballkesir) of Uludağ University, Associate Professor in 1989, in 1995 Professor at Faculty of Education of Dokuz Eylul University. In 2004, Mathematics Professor at Faculty of Science at Muğla University and since 2012, he has been Applied Mathematics Professor at Faculty of Science at Celal Bayar University. 
His main research interests are ordinary and partial differential equations, integral and integro differential -difference equations, delay differential equations and their numerical solutions. Prof. Sezer has been reviewer for numerous influential journals, has published research articles related to differential equations, linear algebra, analytic geometry and calculus; and has been authored over 100 papers. 